Research Article

\title{
Enhancement of the Piezoelectric Cantilever Beam Performance via Vortex-Induced Vibration to Harvest Ocean Wave Energy
}

\author{
Xiaozhen Du, ${ }^{1}$ Yan Zhao, ${ }^{1}$ Guilin Liu $\mathbb{D}^{2},{ }^{2}$ Mi Zhang, ${ }^{1}$ Yu Wang, ${ }^{1}$ and Hong $\mathrm{Yu}^{3}$ \\ ${ }^{1}$ College of Mechanical and Electronic Engineering, Shandong University of Science and Technology, Qingdao 266590, \\ Shandong, China \\ ${ }^{2}$ College of Engineering, Ocean University of China, Qingdao 266100, Shandong, China \\ ${ }^{3}$ College of Science, China University of Petroleum, Qingdao 266580, Shandong, China
}

Correspondence should be addressed to Guilin Liu; liuguilin73@ouc.edu.cn

Received 11 June 2020; Revised 8 July 2020; Accepted 22 July 2020; Published 14 September 2020

Academic Editor: Hong-jun Zhu

Copyright (C) 2020 Xiaozhen Du et al. This is an open access article distributed under the Creative Commons Attribution License, which permits unrestricted use, distribution, and reproduction in any medium, provided the original work is properly cited.

\begin{abstract}
Renewable and sustainable energies exhibit promising performance while serving as the power supply of a wireless sensor especially located in marine waters. Various microgenerators have been developed to harvest wave energy. However, the conversion ability from a dynamic oscillating source of wave is crucial to enhance their effectiveness in practical applications. In this paper, a new piezoelectric converter system is proposed to harvest the kinetic energy from ocean waves. The vortex-induced effect in an air channel enhances the vibration performance, improving the energy harvesting efficiency. The system comprises an oscillating water column (OWC) air chamber, a bluff body, and a piezoelectric piece for electromechanical transduction. The fluid-solid-electric coupling finite element method was used to investigate the relation between the output voltage and geometrical parameters, including the size and position of the piezoelectric cantilever beam, which is based on the user-defined function of the ANSYS. It is found that the bluff body in the outlet channel above the air chamber induced high-frequency vortex shedding vibration. The regular wave rushed into the air chamber with a frequency of $0.285 \mathrm{~Hz}$ and extruded the air across the bluff body in the outlet channel. This incurred the fluctuation of the air pressure and excited the piezoelectric cantilever beam vibration with a high frequency of $233 \mathrm{~Hz}$ in the wake region. Furthermore, a continuous electrical output with a peak voltage of $6.11 \mathrm{~V}$ is generated, which has potential applications for the wireless sensors on the marine buoy.
\end{abstract}

\section{Introduction}

Piezoelectric power generation driven by ocean waves is a promising alternative or self-contained power source for remote wireless sensor nodes [1, 2]. Particularly, energy harvesting from flow-induced vibrations (FIVs) has become a hot topic $[3,4]$. Energy harvesting from FIVs includes generally vortex-induced vibration [5, 6], galloping [7-9], and flutter and wake galloping $[10,11]$. Besides, energy harvesting from wave-induced vibration also calls a lot of attention; e.g., Nan $\mathrm{Wu}$ developed a piezoelectric coupled buoy energy harvester containing several piezoelectric coupled cantilevers attached to a floating buoy structure [12]. This energy harvester can be easily suspended in intermediate and deep ocean for energy harvesting by adjusting the sizes of the floater and sinker. However, it is considerably challenging to harvest this wave energy concentrated in a relatively narrow and lower frequency band. Recently, the piezoelectric generating patch operation frequency has been optimized to improve the energy harvesting efficiency; e.g., Zhao provided an optimized design of the piezoelectric cantilever beam at a low frequency based on theoretical analysis and simulation [13]. Zhang introduced a high-frequency generating piezoelectric beam with a lowfrequency drive beam from an impact rope as a tunable wideband frequency upconversion to harvest the vibration energy of low-frequency environments [14]. Fan proposed a nonlinear piezoelectric energy harvester containing stoppers 
and magnets, causing the operation frequency toward a lower frequency and monostability by modifying the gap between the tip mass and external magnets [15]. Moon expanded the bandwidth of the piezoelectric cantilever beam by tuning a proof mass that caused the difference between the consecutive flexural resonance frequencies of the beam [16]. Therefore, the bandwidth increased by $426.6 \%$ at the same output level and the power improved by $508.5 \%$ when compared with the conventional device. Li M. exploited the nonlinearity and structural coupling effect in vibration energy harvesting to construct piezoelectric harvesters having $\mathrm{X}$-structures characterized by two mounting configurations which meets with ultralow-frequency range of traditional cantilever-based harvesters [17].

The ocean wave frequency of $0.3-1 \mathrm{~Hz}$ is considerably low in comparison with a resonance frequency of several hundred hertz for the piezoelectric patch $[18,19]$. Therefore, generation of high power cannot be achieved by simply synchronizing the movement of the piezoelectric patch with that of the ocean wave. The excitation frequency improves energy conversion efficiency because the piezoelectric generating power increases with an increase in the vibration frequency. Several studies have explored frequency adjustment techniques such as the addition of auxiliary massspring systems [20], the usage of generator arrays [21], and increasing the frequency fluctuation by the vortex shedding effect [22]. Nabavi studied a self-tuning beam-column piezoelectric-based energy harvesting system that can be optimally used as an ocean wave energy harvester when subjected to large wave height and a low frequency [23]. The bimorph piezoelectric generator described by Okada is excited by two pendulums that oscillate with a floating body motion at the ocean wave surface [24]. The pendulums hit the piezoelectric element six times within each wave cycle even though the wave period range is $0.5-1.5 \mathrm{~s}$. The collision duration can be adjusted with the length of the fishing line used to suspend a ball. Viet proposed an innovative design containing a harvester comprising $n$ blades and a stator attached to eight mass-spring-piston-cylinder-piezoelectricity devices [25]. The resonance and force magnification improved the power output of the harvester. Hwang introduced a piezoelectric energy harvesting system in which a magnet is the tip mass at the end of the piezoelectric cantilever structure and a rail with a metal ball is capable of harvesting energy from low-frequency vibrations induced by ocean waves [26]. Murray presented a two-stage piezoelectric energy generator with buoyant structures that interact with the ocean waves as a low-frequency source and excite an array of vibrating piezoelectric elements into higher-frequency resonance, similar to a musician strumming a guitar [27]. Zhang proposed a multi-impact piezoelectric harvester composed of a hung mass and two stiff piezoelectric cantilever beams to enhance the operation performance at a super-low frequency [28]. The harvester was triggered with high-frequency vibrations and the electric power converted from the series of sequential impacts is triple that of a single-impact device in one vibration cycle. Viet developed a variant of the harvester containing four magnetic bar-mass-spring-lever-piezoelectric systems that are symmetrically arranged to transform the low-frequency ocean wave energy into electricity, harnessing high power $[29,30]$.

The natural fluid flows are flat, and a vortex can be generated by inserting a bluff body in the fluid to improve the harvesting efficiency [31-34]. Wang presented a flow-induced piezoelectric energy harvester comprising a diaphragm and a piezoelectric laminate subjected to a distributed and oscillating load in a flow channel with fluctuating pressure [35]. Petrini and Song presented piezoelectric energy harvesters involving vortex-induced vibrations based on air and water flows, respectively [36, 37]. Akaydin used a thin flexible piezoelectric cantilever beam in case of a turbulent wake flow induced by the cylindrical bluff body at a high Reynolds number $(\operatorname{Re}>10,000)$ to facilitate its utilization at high frequencies for energy harvesting [38]. Nguyen arranged tandem double bluff bodies to enhance the pressure fluctuation amplitude on the piezoelectric film denoting a vortex street [22]. Quan Wen simulated the vortex-induced pressure based on different bluff body shapes, including cuboid and cylinder shapes. The pressure of the former was approximately five times that of the latter. A comb-shaped bluff body in the wake can reduce secondary vortex shedding [39]. Yun studied vortex street vibration energy harvesting in airflow induced by running cars; meanwhile, $\mathrm{Hu}$ introduced a vortex-induced piezoelectric sensor to measure the low-speed wind flow [40, 41].

The piezoelectric cantilever beam is easily broken by rigid mechanical shock. Harsh conditions are highly pursued to an ocean wave for obtaining reliable energy conversion technologies. We developed a piezoelectric harvester with an oscillating water column (OWC) in its air chamber. This device converts low-frequency ocean waves into higher frequencies of air pressure fluctuations, triggering the vibration of the piezoelectric cantilever beam, as depicted in Figure 1. The relation between the generating power and the geometrical parameters of the structure was investigated based on fluid-solid-electric coupling by the finite element method. The new energy harvesting system improves the fluctuating frequency and the output voltage amplitude of the piezoelectric cantilever beam induced by the ocean wave.

\section{Methods}

2.1. Structure and Operating Principle. The wave energy harvesting device comprises a semiclosed air chamber and a piezoelectric patch. The air chamber is a semisubmerged vertical OWC converter on the water surface and it is attached to the seafloor with rope. The free surface oscillating motion in the chamber was induced by the incident waves, trapping the air above the free surface of the water to flow through a cylindrical bluff body fixed in the outlet channel and induce vortex shedding. The piezoelectric cantilever beam was positioned in the wake flow region to vibrate with swirling vortices, as presented in Figure 2. When the ocean wave flows into the air chamber, the ocean wave energy is transformed into oscillation kinetic energy at the free water surface of the internal chamber [42]. The air is compressed 


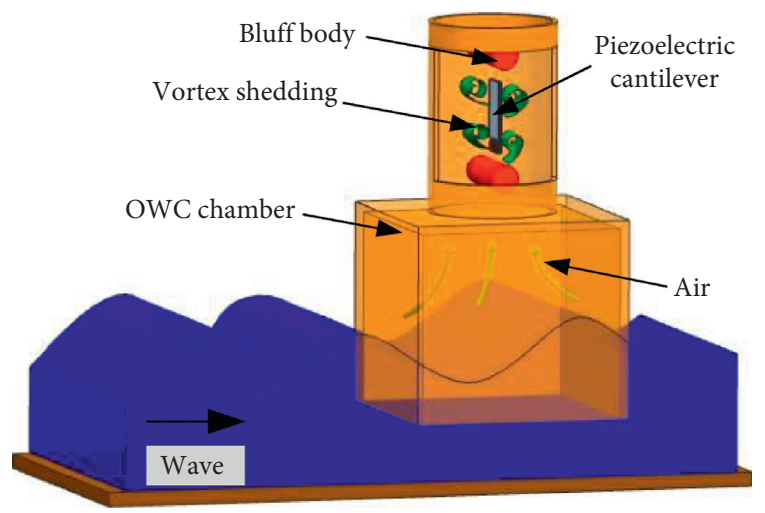

FIGURE 1: Schematic of the piezoelectric cantilever beam generator with OWC harvesting the ocean wave energy.

by the OWC and flows across the bluff body in the exhaust channel with a Reynolds number $\left(40<\operatorname{Re}<3.0 \times 10^{5}\right.$ or $\left.\operatorname{Re}>3.5 \times 10^{6}\right)$. A series of alternating vortices and unsteady pressure distribution of the vortex-induced vibration is generated thereafter in the wake flow region. Subsequently, the low-frequency ocean wave motion is converted to a higher-frequency airflow vortex-excited vibration. The operating frequency range can be adjusted through the gap between the bluff body and the piezoelectric cantilever beam to improve the energy harvesting efficiency.

\subsection{Mathematical Expressions for the Vortex Shedding} Frequency. The equations for the two-phase flow model of air and water were adopted by assuming potential flow and an incompressible fluid to analyze the hydrodynamic behavior of the OWC air chamber on the free ocean wave surface exhibiting a finite water depth in the time domain. According to energy balance and mass conservation in the water tank models of fluid flows, the water surface fluctuations with rising and falling movements compress the air flowing through the channel. Simultaneously, the airflow velocity can be calculated based on the change in the volume of the air chamber.

Further, the small amplitude wave theory was adopted to determine the incident wave energy. The wave oscillation amplitude of the free water surface $\zeta(t)$ can be expressed as

$$
\zeta t=R a \sin (\omega t+\varnothing)
$$

where $R$ is the horizontal distance of the source point from the field point and $a$ is the wave amplitude.

The source point is the point at which the water flows out from the left side of the sink, whereas the field point is the point at which there is no water. The parameters $\omega$ and $\varphi$ denote the natural frequency of the wave and the initial vibration phase of the water surface in the air chamber. Subsequently, the oscillation velocity $v(t)$ of the free water surface can be calculated as a derivative of (1) as follows:

$$
v(t)=\frac{d \zeta}{d t}=R a \omega \cos (\omega t+\varnothing)
$$

The ocean waves entering the air chamber result in a continuous OWC. The air above the water surface flows through the circular air outlet at the top of the chamber. Meanwhile, the air volume changes inside the chamber in a breath cycle of exhaust or suction was obtained as follows

$$
Q_{C}=\frac{2 S}{T} \int_{(\mathrm{n}+1 / 4) T}^{(\mathrm{n}+3 / 4) T} R a \omega \cos (\omega t) \mathrm{d} t,
$$

where $S$ is the horizontal cross-sectional area of the air chamber in Figure 2, $S=L_{C} \times W_{C}$, and $T$ is the wave period.

The airflow $Q_{D}$ in the air passage is equal to the air volume change in the chamber. The quantity of air flowing in the air passage can be calculated as $Q_{\mathrm{D}}=V_{\mathrm{D}} \times S_{1}$, where $V_{\mathrm{D}}$ and $S_{1}$ denote the free water surface oscillation speed and the cross-sectional area of the air outlet in coping of the chamber wall, respectively.

$$
V_{D}=\frac{2 S}{s_{1} T} \int_{(n+1 / 4) T}^{(n+3 / 4) T} R a \omega \cos (\omega t) \mathrm{d} t .
$$

Turbulence or vortex is formed when the exhaust or suction airflow passes the stationary cylindrical bluff body in the outlet channel, whether it is steady or unsteady. A stable periodic frequency of vortex shedding is related to the Reynolds number Re that can be described as follows [43]:

$$
\operatorname{Re}=\frac{V_{D} \times D}{v},
$$

where $D$ is the diameter of the cylindrical bluff body and $v$ is the dynamic viscosity coefficient of the air $\left(1.789 \times 10^{-5} \mathrm{~m}^{2} / \mathrm{s}\right)$.

When $\operatorname{Re}>40$, the sustaining pressure fluctuation produced in the area behind the bluff body is utilized to excite the piezoelectric cantilever beam. The fluctuation frequency related to vortex shedding is described using the diameter of the bluff body $D$, the flow velocity $V_{\mathrm{D}}$, and the Strouhal number $S_{\mathrm{t}}$ as follows [44]:

$$
f_{s}=\frac{S_{t} \times V_{D}}{D}
$$

where $S_{\mathrm{t}}$ is the nondimensional Strouhal number, which can be assumed to be 0.2 for the circular section, and $f_{\mathrm{s}}$ is the fluctuation frequency in case of vortex shedding [36].

The generation efficiency is significantly improved when the piezoelectric cantilever beam vibration resonates with pressure fluctuation. Furthermore, the vortex-excited frequency and the eddy current velocity are optimized and adjusted based on the diameter of the bluff body and the spatial position of the piezoelectric cantilever beam based on the fluid-structure interaction (FSI) simulations using the finite element method.

\section{Numerical Simulation Results and Discussion}

A multiphysical field coupling model was introduced to simulate the performance of the new power generation system involving the fluid-solid coupling of the water and 


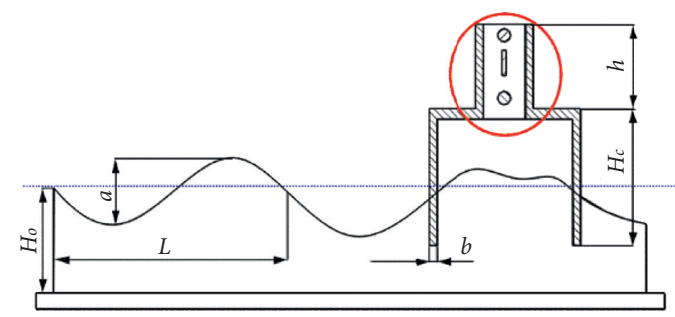

(a)

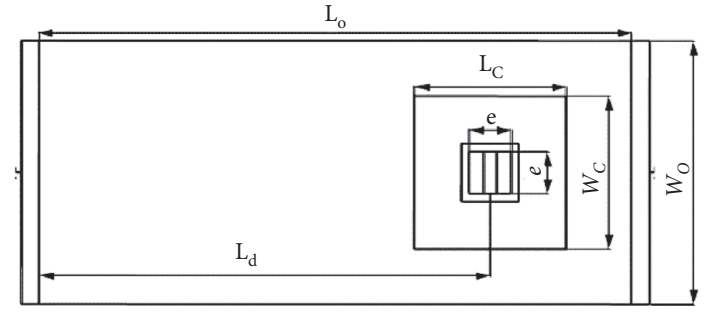

(c)

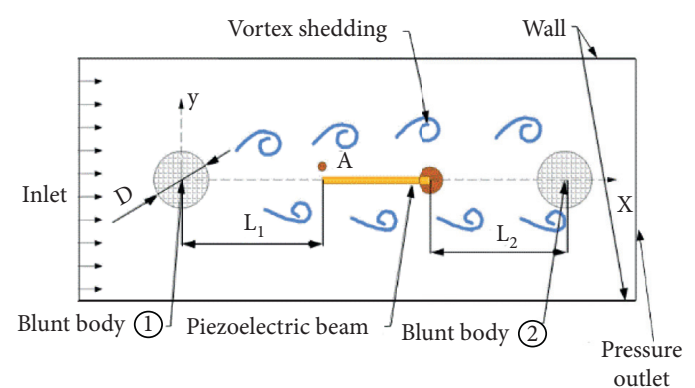

(b)

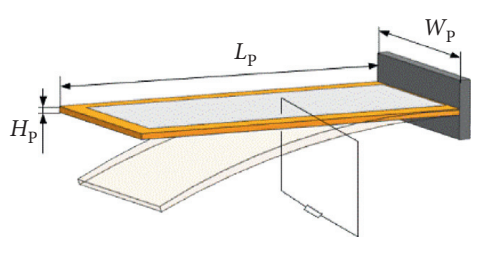

Figure 2: The structure of the piezoelectric wave energy harvester. (a) OWC piezoelectric generator in the water flume as shown with the front and planform view, (b) the vortex shedding in the channel, and (c) piezoelectric cantilever beam.

the chamber as well as the airflow and the cantilever beam and the electromechanical coupling of the piezoelectric material. A three-dimensional (3D) numerical wave flume model of $1: 1$ scale was set up with the computational fluid dynamics (CFD) software to simulate the wave-making and wave energy harvesting processes. Simultaneously, vortex shedding was simulated when the pressed air flows across the bluff body at the outlet channel, as illustrated in Figure 3. Firstly, the air velocity at the air chamber outlet was simulated. Secondly, the variable velocity was incorporated as the initial motion in the user-defined function (UDF) model of the vortex-induced piezoelectric cantilever vibration system to simulate high frequency. Finally, the output voltage on the piezoelectric cantilever beam was obtained by fluid-solid-electric coupling analysis. The effects of several factors about power generation are discussed including the size of the bluff body and spatial position of the piezoelectric cantilever beam in the airflow channel.

3.1. The 3D CFD Numerical Wave Flume Simulation. The 3D numerical wave flume with an oscillating air chamber model is presented in Figure 4. The CFD numerical model solves the Navier-Stokes equation instead of obtaining the potential flow based on the incompressible viscous fluid dynamics continuity hypothesis [45-47]. The specific wave and flume structural parameters are presented in Table 1. The volume of fluid (VOF) method was adopted to track the fluid interface of the air-liquid two-phase flow in the numerical wave flume and determine the volume fraction of fluids (air and water) in each control volume unit as shown in Figure 4(a) [42-44]. The compressed air resulted in continuous vortex shedding by flowing across the bluff body. Simultaneously, the positive and negative air pressures were alternated in the wake region, as depicted in Figure 4(b) with the wave period $(T)$ of $0.4 \mathrm{~s}$.

In the numerical analysis model of the generator, the ocean water depth was set to $4 \mathrm{~m}$ from the free surface of the wave to the bottom of the sea, as illustrated in Figure 2(a). The incident wave period $(T)$ was $3.5 \mathrm{~s}$, and the wave height $(H)$ was $0.8 \mathrm{~m}$. The OWC was placed at $L_{\mathrm{d}}=20 \mathrm{~m}$ from the wave inlet boundary. The air velocity at the outlet was obtained after simulation. As the waves invaded the OWC air chamber in the numerical wave flume, the air velocity gradually increased and periodically stabilized and varied after $5.0 \mathrm{~s}$. The time history of the velocity curve that approximately denotes a cosine wave is displayed in Figure 5. The maximum exhaust and suction velocities of the air are 46.78 and $57.01 \mathrm{~m} / \mathrm{s}$, respectively.

The time history of air velocity includes the exhaust and inhalation states, with the oscillation of the OWC in the chamber. The fitting curve in a cycle of time history $(T=3.5 \mathrm{~s})$ was extracted from Figure 5 to analyze the airflow process presented in Figure 6 . The velocity values located above the zero line of the coordinates from 0 to $2 \mathrm{~s}$ represent the exhausted state, with a corresponding inhalation state being observed from 2 to $3.5 \mathrm{~s}$. The piezoelectric cantilever beams were vibrated by the air pressure motions, as depicted in Figure 6. The variable velocity is the inlet parameter of the UDF numerical simulation obtained from the equation by curve fitting with the MATLAB software. This was simulated in a step-by-step manner for the flow solid coupling fields of the water and chamber, the high-pressure air, and the piezoelectric cantilever beam.

3.2. Piezoelectric Generation Simulation with Vortex Shedding. Two bluff bodies were set up at the inlet and outlet air channel to harvest the exhaust and inhalation air 


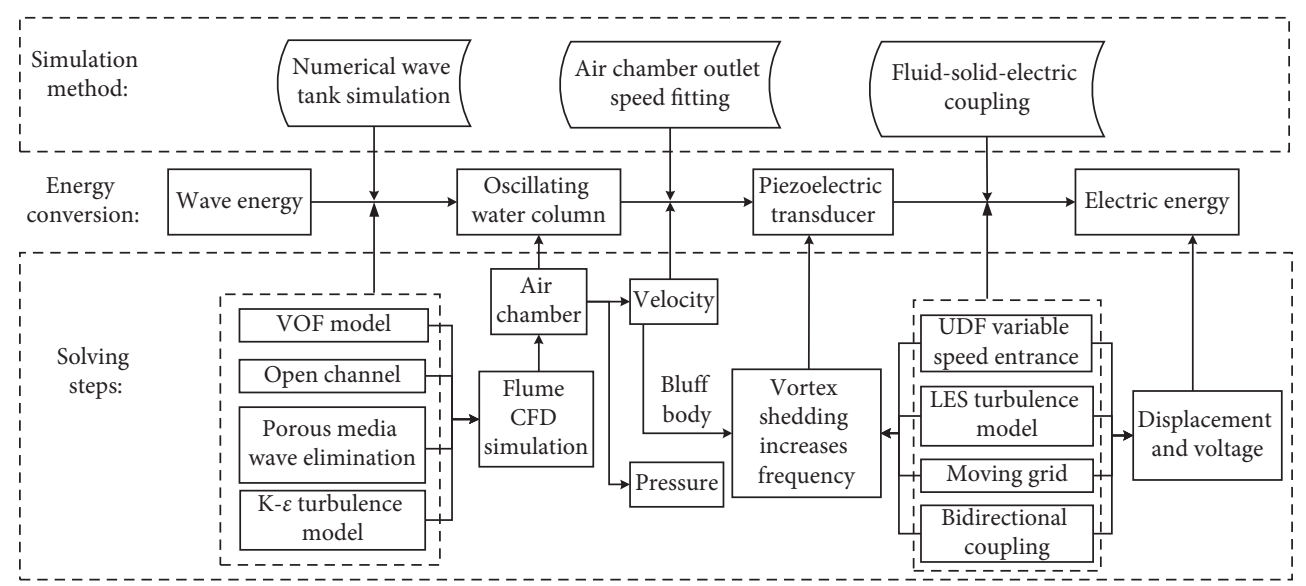

FigURE 3: Schematic of the simulation process for the piezoelectric generator system.

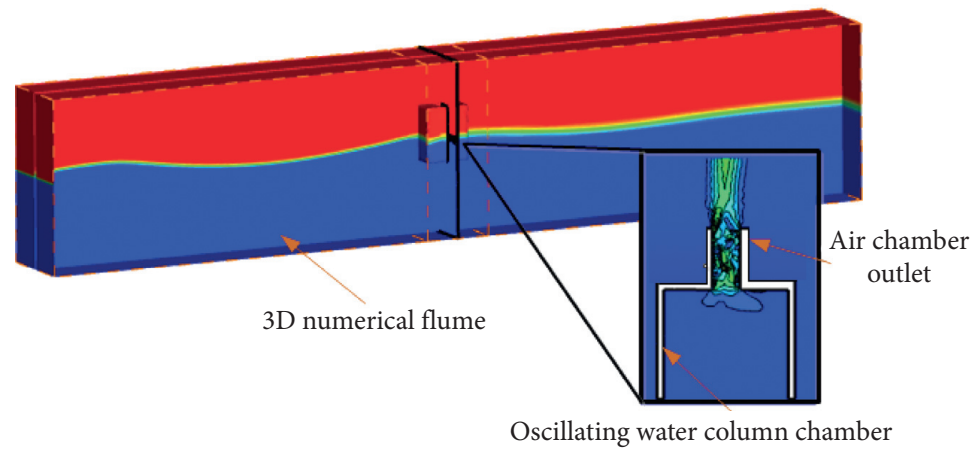

(a)

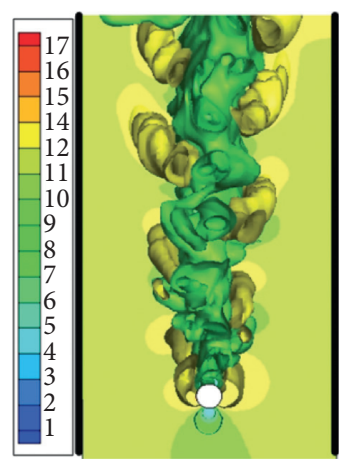

(b)

Figure 4: Simulation of the 3D numerical wave energy harvesting system. (a) Volume fraction of the flume and chamber. (b) Vortex shedding diagram at the outlet of air chamber $(T=0.4 \mathrm{~s})$.

TABLE 1: Parameters of the 3D numerical model of the wave energy harvester.

\begin{tabular}{lcc}
\hline Components & \multicolumn{1}{c}{ Dimensions } \\
\hline & Length $\left(L_{0} / \mathrm{m}\right)$ & 40 \\
Numerical flume & Width $\left(W_{0} / \mathrm{m}\right)$ & 4 \\
& Still water depth $\left(H_{0} / \mathrm{m}\right)$ & 4 \\
& Wave height $(H / \mathrm{m})$ & 0.8 \\
& Wave period $(T / \mathrm{s})$ & 3.5 \\
& Length $\left(L_{\mathrm{C}} / \mathrm{m}\right)$ & 2 \\
OWC chamber & Width $\left(W_{\mathrm{C}} / \mathrm{m}\right)$ & 2 \\
& Height $\left(H_{\mathrm{C}} / \mathrm{m}\right)$ & 1.8 \\
& Wall thickness $(b / \mathrm{m})$ & 0.1 \\
& Air outlet $\left(\mathrm{e} \times \mathrm{e} \times h / \mathrm{m}^{3}\right)$ & $0.3 \times 0.3 \times 0.7$ \\
Piezoelectric cantilever & Length $\left(L_{\mathrm{P}} / \mathrm{m}\right)$ & 0.05 \\
& Width $\left(W_{\mathrm{P}} / \mathrm{m}\right)$ & 0.03 \\
& Thickness $\left(H_{\mathrm{P}} / \mathrm{m}\right)$ & 0.001 \\
\hline
\end{tabular}

kinetic energy, respectively, as shown in Figure 2(c). The finite volume method was adopted to model and solve the pressure-velocity coupling term and the large-scale turbulence structure to analyze the vortex aerodynamic characteristics $[40,48]$. Further, a sublattice model was constructed

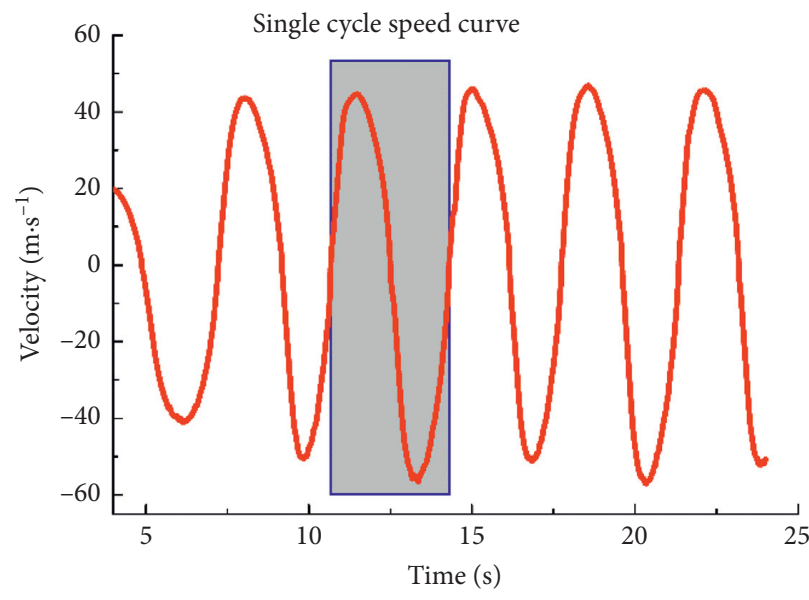

FIGURE 5: Time history of the air velocity at the outlet of the chamber.

for the small-scale isotropic large-eddy simulation turbulence model to precisely distinguish the vortex-excited structure in the flow field. 


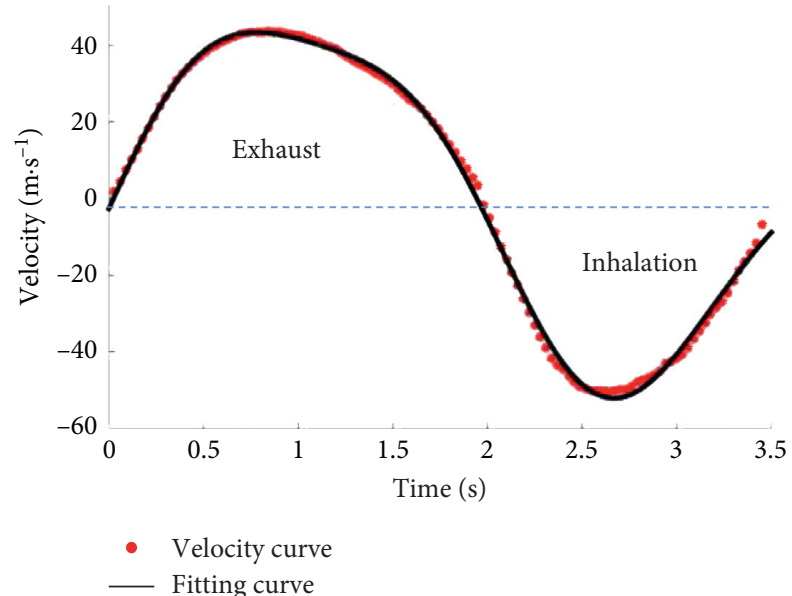

Figure 6: The airflow velocity fitting curve with the oscillation of the OWC.

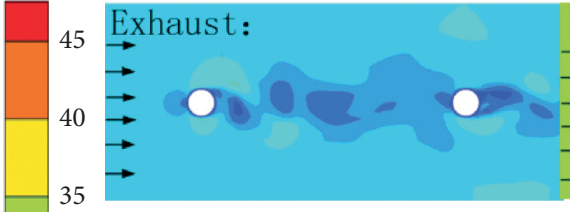

(a)

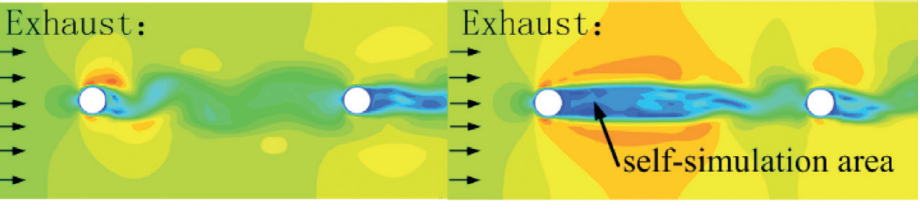

(b)

(c)

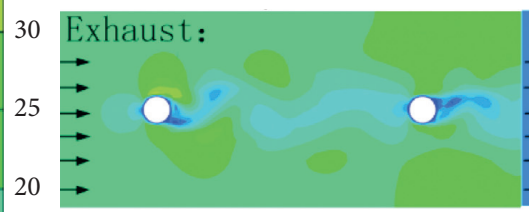

(d)

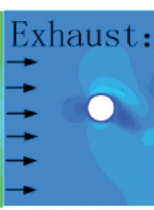

15

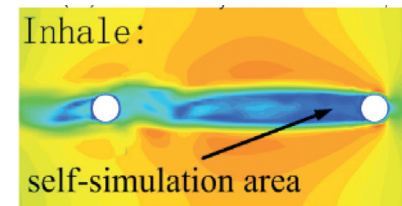

(g)

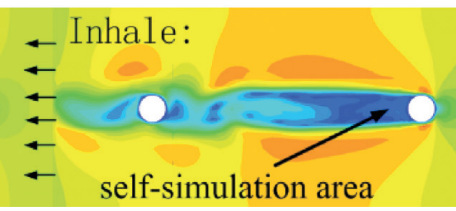

(h)

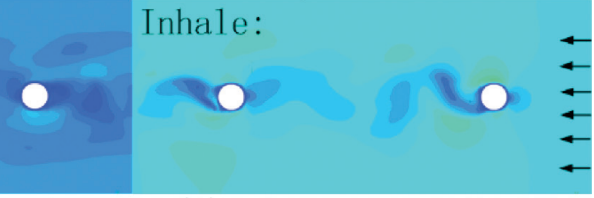

(f)
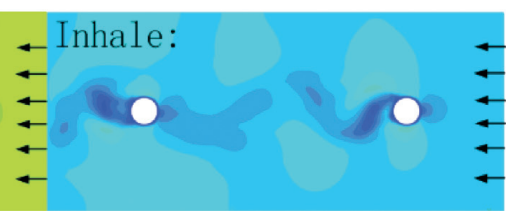

(i)

Figure 7: The air velocity cloud images across the bluff body with time. (a) $t=0.2 \mathrm{~s}, v=15.68 \mathrm{~m} / \mathrm{s}$. (b) $t=0.6 \mathrm{~s}, v=40.99 \mathrm{~m} / \mathrm{s}$. (c) $t=1 \mathrm{~s}$, $v=45.34 \mathrm{~m} / \mathrm{s}$. (d) $t=1.4 \mathrm{~s}, v=32.81 \mathrm{~m} / \mathrm{s}$. (e) $t=1.8, v=14.19 \mathrm{~m} / \mathrm{s}$. (f) $t=2.2 \mathrm{~s}, v=19.31 \mathrm{~m} / \mathrm{s}$. (g) $t=2.6 \mathrm{~s}, \mathrm{v}=49.85 \mathrm{~m} / \mathrm{s}$. (h) $t=3 \mathrm{~s}, v=41.83 \mathrm{~m} / \mathrm{s}$. (i) $t=3.4 \mathrm{~s}, v=20.47 \mathrm{~m} / \mathrm{s}$.

The unsteady flow induced by the bluff body was simulated using an integration time step of $3.5 \mathrm{~s}$. Reynolds number $(\mathrm{Re})$ constantly changes with the cosine variation of air velocity at the outlet based on (5). Simultaneously, the vortex shedding characteristics also vary with Re. The velocities and vortex shedding distributions captured using the CFD simulation model are presented in Figure 7 according to the air velocity time history. Re becomes 35058 at $0.2 \mathrm{~s}$ when the air flowed across the circular bluff body $(D=40 \mathrm{~mm})$ with an instantaneous velocity of $15.68 \mathrm{~m} / \mathrm{s}$. At the bluff body surface, the boundary layer of airflow was laminar before waking and transforming into a subcritical region of turbulence $\left(300<\operatorname{Re}<2.5 \times 10^{5}\right)$ behind the bluff body, as exhibited in Figure 7(a). After 1.0 s, Re increases to become greater than $10^{5}$, with the subcritical region becoming supercritical $\left(2.5 \times 10^{5}<\operatorname{Re}<3 \times 10^{5}\right)$. The flow separation point moves toward the tail, and the vortex

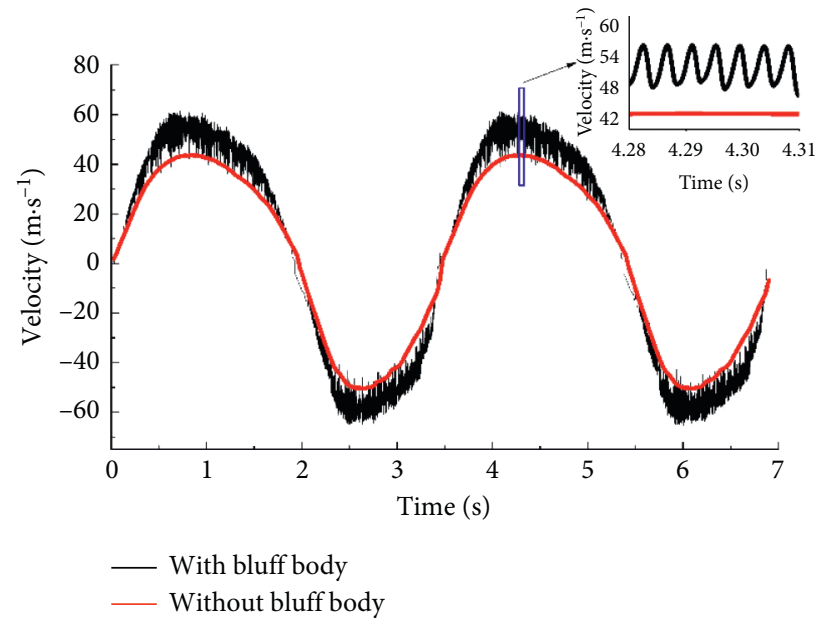

FIgURE 8: Comparison of the velocities at the outlet channel with and without the bluff body. 


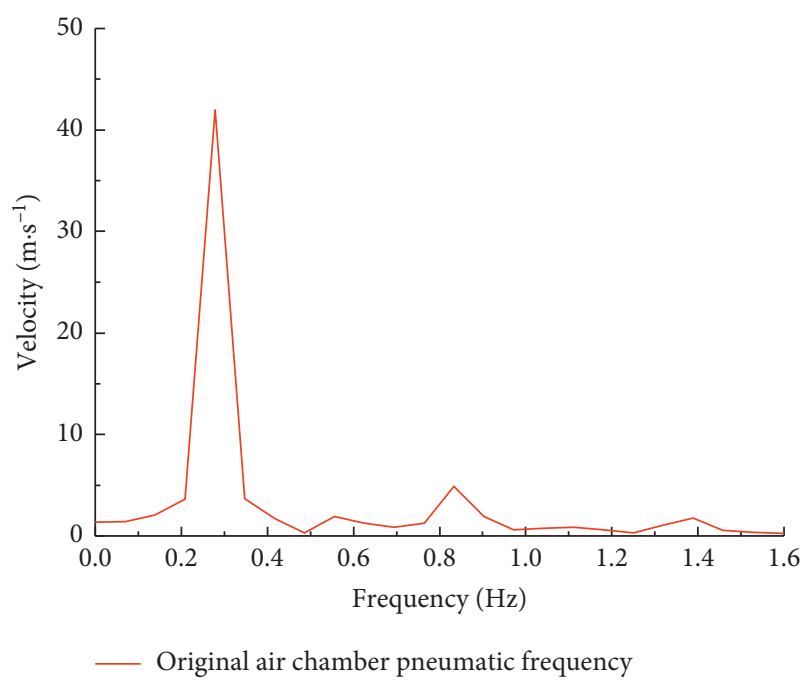

(a)

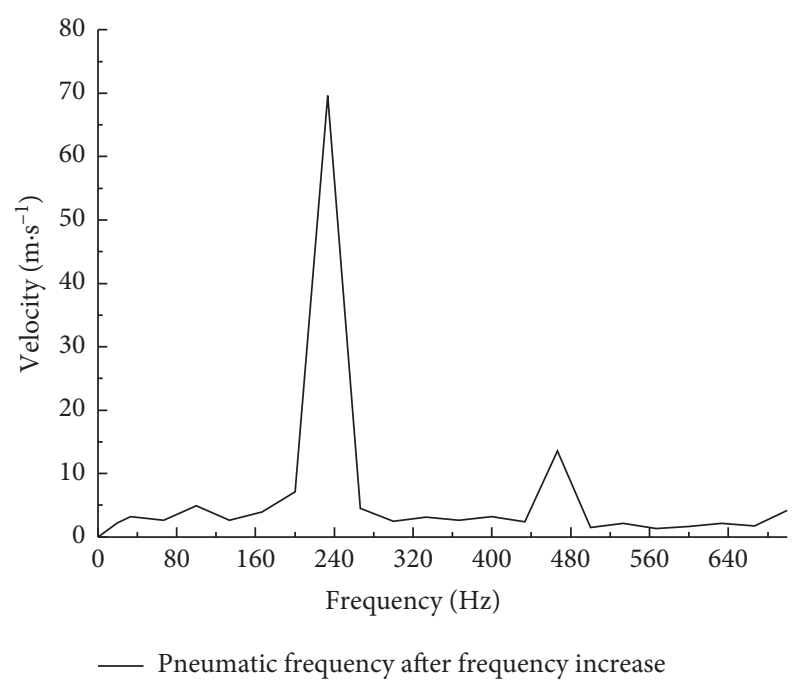

(b)

FIGURE 9: Pneumatic frequency in the channel. (a) The original frequency with OWC. (b) The shedding pneumatic frequencies.

Table 2: Materials of the piezoelectric vibrator.

\begin{tabular}{lcccc}
\hline Material & Young's modulus $10^{10}\left(\mathrm{~N} / \mathrm{m}^{2}\right)$ & Density $10^{3}\left(\mathrm{~kg} / \mathrm{m}^{3}\right)$ & Poisson's ratio $\sigma$ & Mechanical quality coefficient $\left(Q_{\mathrm{m}}\right)$ \\
\hline Brass & 11.2 & 8780 & 0.35 & - \\
PZT-4 & 5.6 & 7500 & 0.36 & 70 \\
\hline
\end{tabular}

TABLE 3: The properties of PZT-4.

\begin{tabular}{|c|c|c|c|c|c|c|c|c|c|c|}
\hline \multicolumn{6}{|c|}{ Toughness constant $(1 / \mathrm{Y}) 10^{-12}\left(\mathrm{~m}^{2} / \mathrm{N}\right)$} & \multicolumn{3}{|c|}{$\begin{array}{l}\text { Piezoelectric constant } 10^{-12} \\
(\mathrm{C} / \mathrm{N})\end{array}$} & \multicolumn{2}{|c|}{$\begin{array}{c}\text { Relative dielectric } \\
\text { constant }\end{array}$} \\
\hline$S_{11}$ & $S_{33}$ & $S_{44}$ & $S_{12}$ & $S_{13}$ & $S_{66}$ & $D_{31}$ & $D_{33}$ & $D_{15}$ & $\varepsilon_{11} / \varepsilon_{0}$ & $\varepsilon_{33} / \varepsilon_{0}$ \\
\hline 0.5 & 20.7 & 43.5 & -4.78 & -8.45 & -42.6 & -186 & 670 & 660 & 3130 & 3400 \\
\hline
\end{tabular}
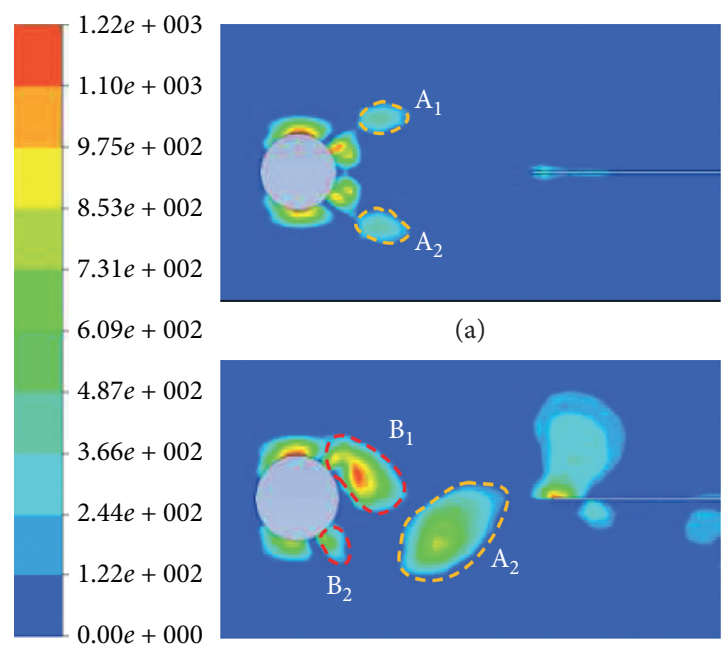

(a)

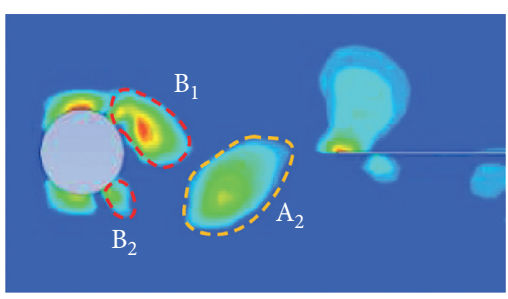

(c)

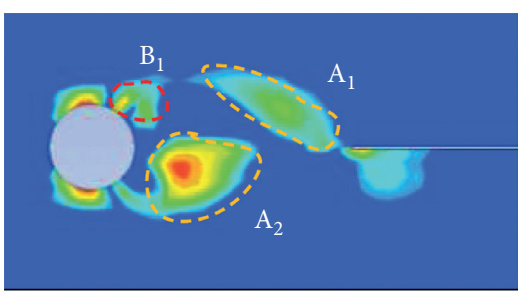

(b)

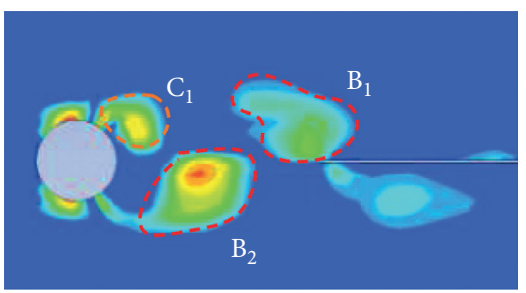

(d)

Figure 10: Vorticity cloud map of piezoelectric cantilever beam with vortex vibration. (a) $t=0.12 \mathrm{~s}$. (b) $t=0.24 \mathrm{~s}$. (c) $t=0.36 \mathrm{~s}$. (d) $t=0.48 \mathrm{~s}$.

shedding area exhibiting turbulence at the wake is a narrow self-simulation area, as shown in Figure $7(\mathrm{c})$. The vortex shedding becomes chaotic and loses the capability to excite the piezoelectric cantilever beam. Once Re reduces with the air velocities to the subcritical region, large-scale vortex shedding reappears in the wake. The same vortex shedding 


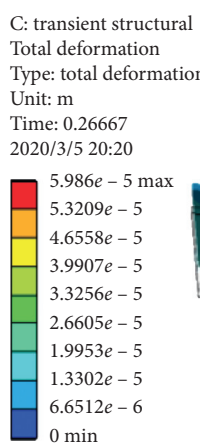

Total deformation

Type: total deformation

Unit: $\mathrm{m}$

Time: 0.8

2020/3/5 20:21

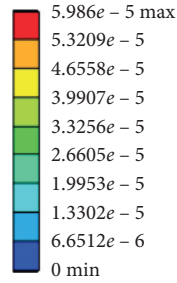

min

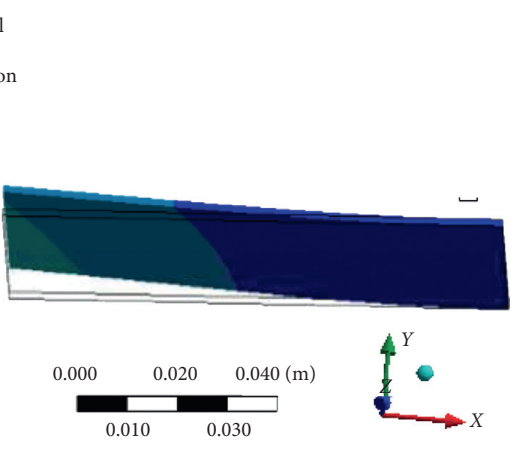

(a)

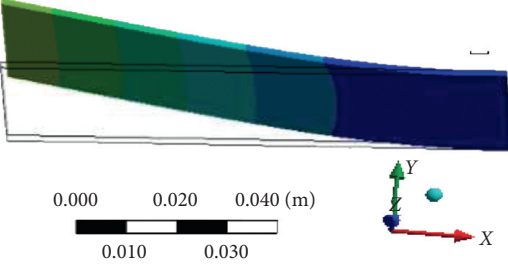

(c)

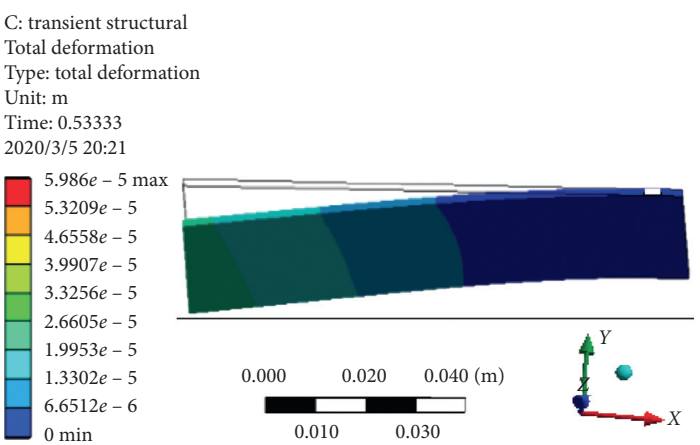

(b)

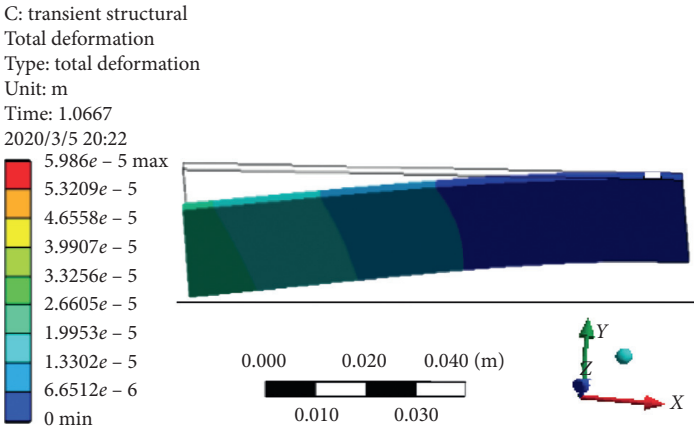

(d)

Figure 11: Deformation of the piezoelectric cantilever beam. (a) $t=0.267 \mathrm{~s}$. (b) $t=0.533 \mathrm{~s}$. (c) $t=0.800 \mathrm{~s}$. (d) $t=1.067 \mathrm{~s}$.

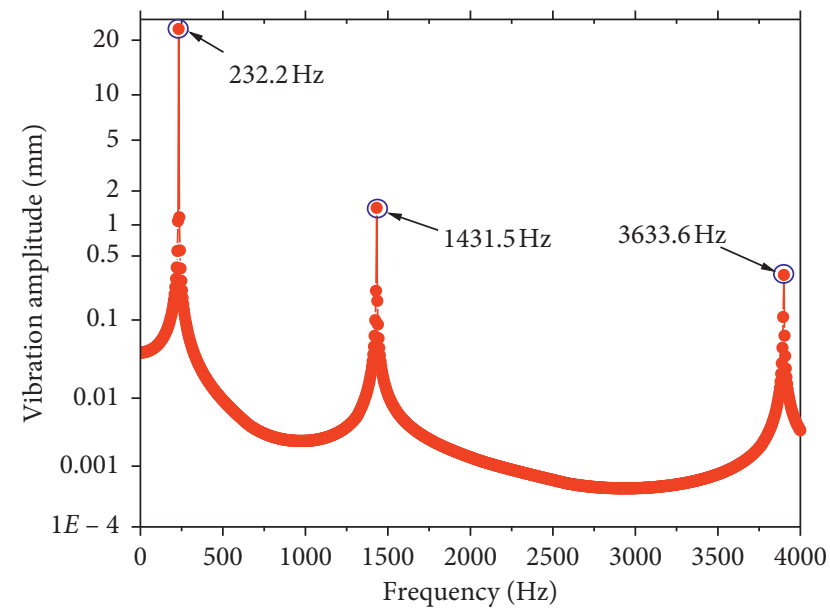

FIGURE 12: The inherent frequency of piezoelectric cantilever beam.

variety process occurs at the inhalation state from 2 to $3.5 \mathrm{~s}$, as shown in Figures 7(f)-7(i). Unsteady flow is induced by the OWC. An approximate cosine form, continuous vortex, and fluctuant pressures produced in the hindrance wake region.

The instantaneous velocity curve at point $A$ in Figure 2(b) is displayed in Figure 8. Air velocity was induced by the cylinder bluff body with sizes of $D=40 \mathrm{~mm}$, $L 1=140 \mathrm{~mm}$, and $L 2=140 \mathrm{~mm}$. The exhaust and inhalation velocities in the channel were significantly improved during two wave cycles in comparison to those without the bluff body. The maximum suction and exhaust velocities

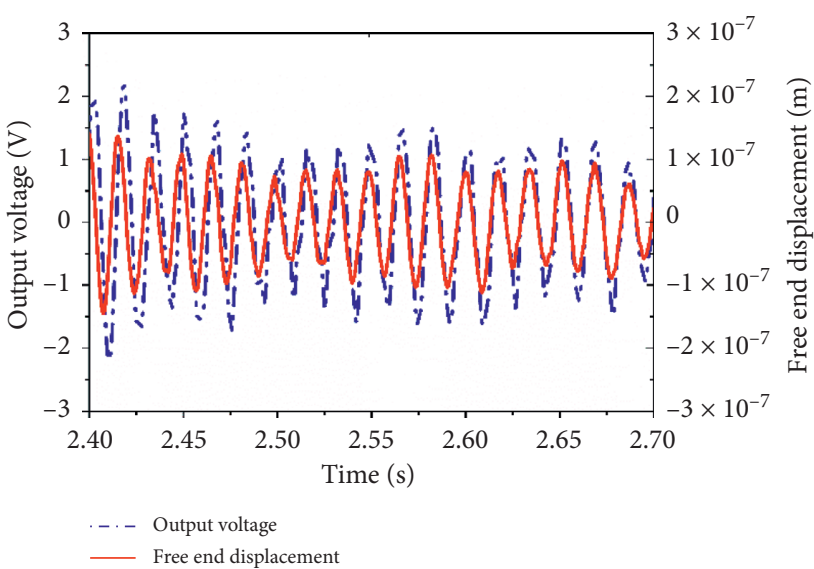

FIGURE 13: Open-circuit voltage and free end displacement on the piezoelectric cantilever beam $((D)=40 \mathrm{~mm})$.

increased from 46.78 and $57.01 \mathrm{~m} / \mathrm{s}$ to 60.92 and $63.84 \mathrm{~m} / \mathrm{s}$, respectively. This resulted in high-frequency fluctuations in velocity for vibration energy harvesting.

Accumulation of waves in the air chamber created an OWC with a wave frequency of $0.285 \mathrm{~Hz}$, as shown in Figure 9(a). The first shedding pneumatic frequency of $233 \mathrm{~Hz}$ was extracted from the fluctuating airflow observed behind the bluff body by fast Fourier transform (FFT), as illustrated in Figure 9(b). The results denote that the vortex vibration with the bluff body in the outlet air channel transforms the low-frequency wave motion into a highfrequency air vortex vibration, improving the power 


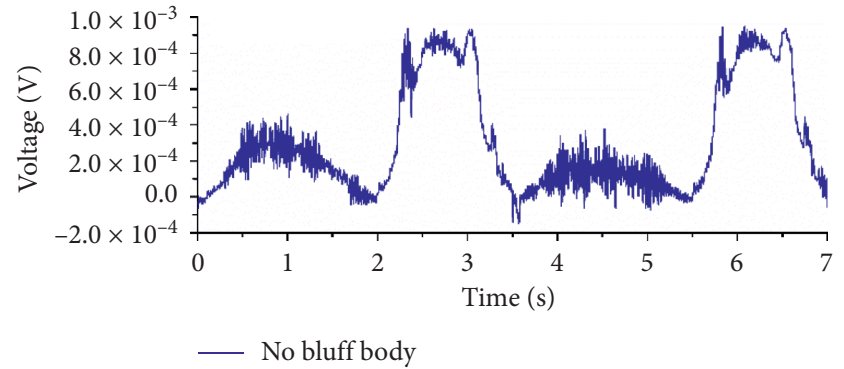

(a)

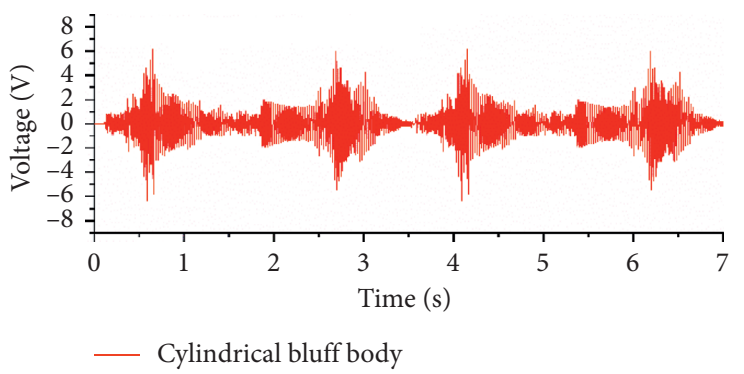

(b)

Figure 14: The voltages on the piezoelectric cantilever beam.

generation efficiency of the piezoelectric element based on the fluctuating air pressure.

As shown in Figure 2(c), a piezoelectric cantilever beam was set up in the outlet channel to harvest the air kinetic energy in the channel. The piezoelectric generator and material parameters are presented in Tables 2 and 3. The brass and PZT-4 materials were selected as the cantilever beam substrate and energy conversion element, respectively.

The ANSYS simulation model sets the interface of air pressure and piezoelectric cantilever beam with solid elements. The symbols of A, B, and C in Figure 10 illustrate the airflow vortex formation and shedding processes. The piezoelectric cantilever beam vibrates in the wake flow region as shown in Figure 10 and the deformations are shown in Figure 11.

The piezoelectric cantilever beam performs with the inherent frequency as shown in Figure 12 which is simulated with ANSYS. The piezoelectric cantilever beam synchronously varies with the aerodynamic force in the wake of the bluff body; the output voltages and free end displacements are presented in Figure 13.

The output open-circuit voltages on the piezoelectric cantilever beams are presented in Figure 14. The peak voltage is increased by a factor of 6000 compared to no bluff body in the outlet air channel with OWC wave energy harvest structure.

3.3. The Generation System Analysis. In this study, the influence of the bluff body was examined, which related to the size of the circumfluence area and the output voltage distribution near the bluff body. The cylindrical bluff body was set in the outlet channel with serial diameters of 40 and $50 \mathrm{~mm}$ to create vortex-induced vibration. The dimensions of the piezoelectric cantilever beam are $50 \mathrm{~mm} \times 30 \mathrm{~mm} \times 1 \mathrm{~mm}$. Further, the spatial position of the beam in the wake region was analyzed and compared with the CFD multiphasic fields. The simulation results exhibit variable vortex shedding flow speeds ranging from 0 to $57.01 \mathrm{~m} / \mathrm{s}$. The distance between the bluff body and the free end of the beam ranges from 80 to $240 \mathrm{~mm}$.

The responses of the output voltages with different locations $\left(L_{1}\right)$ and diameters of the cylindrical bluff body during the exhaust process are depicted in Figure 15. The maximum voltage is approximately $6.8 \mathrm{~V}$ when $L_{1}=200 \mathrm{~mm}$

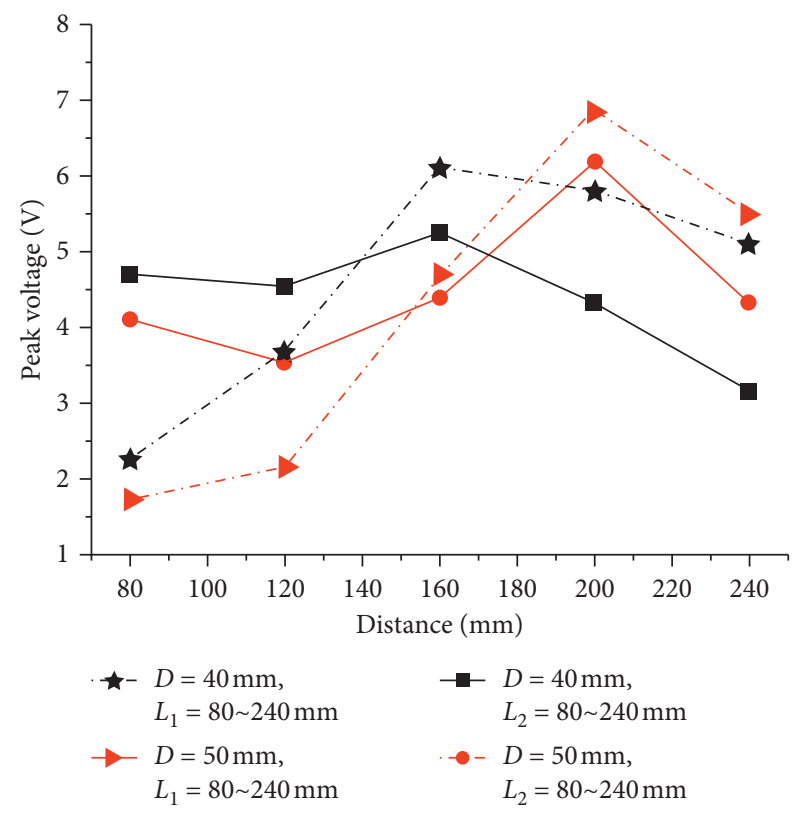

FIGURE 15: The peak open-circuit voltage relative to the fixed spots and diameters of bluff body.

and $D=50 \mathrm{~mm}$. The plot denotes that the voltage decreases when the piezoelectric cantilever beam is located at a distance more than four times the diameter of the bluff body. Meanwhile, the voltage gradually decreases with reducing distance from the bluff body. The maximum peak voltage can be observed when $L_{1}=160 \mathrm{~mm}$ for a bluff body diameter of $40 \mathrm{~mm}$. The corresponding optimal energy acquisition spots exist in the bluff body wake regions for the exhausted state at approximately $L_{1}=4 D$. The same spot also appeared in Figure 15 during the inhalation process.

The peak voltage changes with the distance $\left(L_{1}\right.$ or $\left.L_{2}\right)$ in case of similar bluff body diameters because the vortex excitation is intense at the optimal energy acquisition spot. It will disappear at a remote distance from the fluff body because the cantilever beam is in the vortex attenuation zone. On the contrary, if the cantilever beam locates at a smaller space near the bluff body, no airflow fluctuation in the region is experienced and the piezoelectric cantilever beam vibrates weakly. Therefore, the piezoelectric cantilever requires stability in the optimal wake region with a strong vortex to generate electric power. 


\section{Conclusions}

This study presented a new piezoelectric generation system with an OWC for ocean wave energy harvesting. Further, a simulation model was established to predict the dynamic response of the wave energy harvesting device. The power generation solution simulates with the air-liquid-electromechanical coupling model. In the air chamber, the velocity of the high-frequency airflow in the outlet channel significantly improved with the vortex vibration. The maximum exhaust and suction air velocities increased from 57.01 to $63.84 \mathrm{~m} / \mathrm{s}$ and from 46.78 to $60.92 \mathrm{~m} / \mathrm{s}$, respectively. And the vortex-induced vibration frequency is about $233 \mathrm{~Hz}$ in the air tunnel. The high-frequency vortex aerodynamics excite the piezoelectric cantilever beam to output the maximum opencircuit peak voltage of $6.11 \mathrm{~V}$. The results denoted that the bluff body at the air chamber outlet enhanced the vibration and improved the OWC wave energy harvesting system for the generated electric power. Further, an optimal energy-enhancing region related to the appearance of the vortex is evident in the wake region of the bluff body. The diameter of the bluff body and the spatial location of the piezoelectric cantilever beam will improve the wave energy harvesting.

\section{Data Availability}

The data used to support the findings of this study are available from the corresponding and first author upon request.

\section{Conflicts of Interest}

The authors declare that they have no conflicts of interest.

\section{Acknowledgments}

This research was supported by the National Natural Science Foundation of China (21105234) and the Natural Science Foundation of Shandong Province (ZR2016EEM23).

\section{References}

[1] H. Mutsuda, Y. Tanaka, R. Patel, Y. Doi, Y. Moriyama, and Y. Umino, "A painting type of flexible piezoelectric device for ocean energy harvesting," Applied Ocean Research, vol. 68, pp. 182-193, 2017.

[2] H. Mutsuda, Y. Tanaka, R. Patel, and Y. Doi, "Harvesting flow-induced vibration using a highly flexible piezoelectric energy device," Applied Ocean Research, vol. 68, pp. 39-52, 2017.

[3] J. Wang, L. Geng, L. Ding, H. Zhu, and D. Yurchenko, "The state-of-the-art review on energy harvesting from flow-induced vibrations," Applied Energy, vol. 267, p. 114902, 2020.

[4] J. Wang, S. Gu, C. Zhang et al., "Hybrid wind energy scavenging by coupling vortex-induced vibrations and galloping," Energy Conversion and Management, vol. 213, Article ID 112835, 2020.

[5] H. Zhu, W. Liu, and T. Zhou, "Direct numerical simulation of the wake adjustment and hydrodynamic characteristics of a circular cylinder symmetrically attached with fin-shaped strips," Ocean Engineering, vol. 195, Article ID 106756, 2020.

[6] H. Zhu, G. Li, and J. Wang, "Flow-induced vibration of a circular cylinder with splitter plates placed upstream and downstream individually and simultaneously," Applied Ocean Research, vol. 97, Article ID 102084, 2020.

[7] J. Wang, S. Zhou, Z. Zhang, and D. Yurchenko, "High-performance piezoelectric wind energy harvester with Y-shaped attachments," Energy Conversion and Management, vol. 181, no. 2, pp. 645-652, 2019.

[8] J. Wang, L. Tang, L. Zhao, and Z. Zhang, "Efficiency investigation on energy harvesting from airflows in HVAC system based on galloping of isosceles triangle sectioned bluff bodies," Energy, vol. 172, pp. 1066-1078, 2019.

[9] K. Yang, J. Wang, and D. Yurchenko, "A double-beam piezomagneto-elastic wind energy harvester for improving the galloping-based energy harvesting," Applied Physics Letters, vol. 115, no. 19, Article ID 193901, 2019.

[10] J. Wang, W. Zhao, Z. Su, G. Zhang, P. Li, and D. Yurchenko, "Enhancing vortex-induced vibrations of a cylinder with rod attachments for hydrokinetic power generation," Mechanical Systems and Signal Processing, vol. 115, Article ID 106912, 2020.

[11] G. Hu, J. Wang, Z. Su, G. Li, H. Peng, and K. C. S. Kwok, "Performance evaluation of twin piezoelectric wind energy harvesters under mutual interference," Applied Physics Letters, vol. 115, no. 7, p. 73901, 2019.

[12] N. Wu, Q. Wang, and X. Xie, "Ocean wave energy harvesting with a piezoelectric coupled buoy structure," Applied Ocean Research, vol. 50, pp. 110-118, 2015.

[13] Q. Zhao, Y. Liu, L. Wang, H. Yang, and D. Cao, "Design method for piezoelectric cantilever beam structure under low frequency condition," International Journal of Pavement Research and Technology, vol. 11, no. 2, pp. 153-159, 2018.

[14] J. Zhang and L. Qin, "A tunable frequency up-conversion wideband piezoelectric vibration energy harvester for lowfrequency variable environment using a novel impact- and rope-driven hybrid mechanism," Applied Energy, vol. 240, pp. 26-34, 2019.

[15] K. Fan, Q. Tan, H. Liu, Y. Zhang, and M. Cai, "Improved energy harvesting from low-frequency small vibrations through a monostable piezoelectric energy harvester," $M e$ chanical Systems and Signal Processing, vol. 117, pp. 594-608, 2019.

[16] K. Moon, J. Choe, H. Kim, D. Ahn, and J. Jeong, "A method of broadening the bandwidth by tuning the proof mass in a piezoelectric energy harvesting cantilever," Sensors and Actuators A: Physical, vol. 276, pp. 17-25, 2018.

[17] M. Li and X. Jing, "Novel tunable broadband piezoelectric harvesters for ultralow-frequency bridge vibration energy harvesting," Applied Energy, vol. 255, Article ID 113829, 2019.

[18] W. H. Munk, "Origin and generation of waves," Scripps Institution of Oceannography La Jolla Calif, San Diego, CA, USA, No. SIO-REF-51-57, 1951.

[19] U. Khan and S.-W. Kim, "Triboelectric nanogenerators for blue energy harvesting," ACS Nano, vol. 10, no. 7, pp. 6429-6432, 2016.

[20] G. Wang, W.-H. Liao, B. Yang, X. Wang, W. Xu, and X. Li, "Dynamic and energetic characteristics of a bistable piezoelectric vibration energy harvester with an elastic magnifier," Mechanical Systems and Signal Processing, vol. 105, pp. 427446, 2018.

[21] J.-Q. Liu, H.-B. Fang, Z.-Y. Xu et al., "A MEMS-based piezoelectric power generator array for vibration energy 
harvesting," Microelectronics Journal, vol. 39, no. 5, pp. 802-806, 2008.

[22] H. D. T. Nguyen, H. T. Pham, and D. A. Wang, "A miniature pneumatic energy generator using Karman vortex street," Journal of Wind Engineering and Industrial Aerodynamics, vol. 116, pp. 40-48, 2013.

[23] S. F. Nabavi, A. Farshidianfar, and A. Afsharfard, "Novel piezoelectric-based ocean wave energy harvesting from offshore buoys," Applied Ocean Research, vol. 76, pp. 174-183, 2018.

[24] N. Okada, S. Yabe, H. Fujimoto, and M. Murai, Experiments on Floating Wave-Power Generation Using Piezoelectric Elements and Pendulums in the Water Tank, IEEE, Piscataway, NJ, USA, 2012.

[25] N. V. Viet, M. Al-Qutayri, K. M. Liew, and Q. Wang, “An octo-generator for energy harvesting based on the piezoelectric effect," Applied Ocean Research, vol. 64, pp. 128-134, 2017.

[26] W. S. Hwang, J. H. Ahn, S. Y. Jeong et al., "Design of piezoelectric ocean-wave energy harvester using sway movement," Sensors and Actuators A: Physical, vol. 260, pp. 191-197, 2017.

[27] R. Murray and J. Rastegar, "Novel two-stage piezoelectricbased ocean wave energy harvesters for moored or unmoored buoys," in Proceedings of the Active and Passive Smart Structures and Integrated Systems 2009, p. 7288, San Diego, CA, USA, March 2009.

[28] Y. Zhang and C. S. Cai, "A retrofitted energy harvester for low frequency vibrations," Smart Materials and Structures, vol. 21, Article ID 075007, 2012.

[29] N. V. Viet and Q. Wang, "Ocean wave energy pitching harvester with a frequency tuning capability," Energy, vol. 162, pp. 603-617, 2018.

[30] N. V. Viet, X. D. Xie, K. M. Liew, N. Banthia, and Q. Wang, "Energy harvesting from ocean waves by a floating energy harvester," Energy, vol. 112, pp. 1219-1226, 2016.

[31] H. Zhu, J. Yao, Y. Ma, H. Zhao, and Y. Tang, "Simultaneous CFD evaluation of VIV suppression using smaller control cylinders," Journal of Fluids and Structures, vol. 57, pp. 66-80, 2015.

[32] H. Zhu and J. Yao, "Numerical evaluation of passive control of VIV by small control rods," Applied Ocean Research, vol. 51, pp. 93-116, 2015.

[33] G. Yao, H. Wang, C. Yang, and L. Wen, "Research and design of underwater flow-induced vibration energy harvester based on Karman vortex street," Modern Physics Letters B, vol. 31, no. 7, Article ID 1750076, 2017.

[34] D. A. Wang, H. T. Pham, C. W. Chao, and J. M. Chen, "A piezoelectric energy harvester based on pressure fluctuations in kármán vortex street," in Proceedings of the World Renewable Energy Congress-Sweden, Linköping; Sweden, May 2011.

[35] D.-A. Wang and K.-H. Chang, "Electromagnetic energy harvesting from flow induced vibration," Microelectronics Journal, vol. 41, no. 6, pp. 356-364, 2010.

[36] F. Petrini and K. Gkoumas, "Piezoelectric energy harvesting from vortex shedding and galloping induced vibrations inside HVAC ducts," Energy and Buildings, vol. 158, pp. 371-383, 2018.

[37] R. Song, X. Shan, F. Lv, and T. Xie, “A study of vortex-induced energy harvesting from water using PZT piezoelectric cantilever with cylindrical extension," Ceramics International, vol. 41, pp. S768-S773, 2015.
[38] H. D. Akaydın, N. Elvin, and Y. Andreopoulos, "Wake of a cylinder: a paradigm for energy harvesting with piezoelectric materials," Experiments in Fluids, vol. 49, no. 1, pp. 291-304, 2010.

[39] Q. Wen, R. Schulze, D. Billep, T. Otto, and T. Gessner, "Modeling and optimization of a vortex induced vibration fluid kinetic energy harvester," Procedia Engineering, vol. 87, pp. 779-782, 2014.

[40] S.-M. Yun and C. Kim, "The vibrating piezoelectric cantilevered generator under vortex shedding excitation and voltage tests," International Journal of Precision Engineering and Manufacturing, vol. 17, no. 12, pp. 1615-1622, 2016.

[41] J. Hu, H. Peng, T. Liu, X. Yao, H. Wu, and P. Lu, "A flow sensing method of power spectrum based on piezoelectric effect and vortex-induced vibrations," Measurement, vol. 131, pp. 473-481, 2019.

[42] E. Medina-Lopez, A. G. L. Borthwick, and A. Moñino, "Analytical and numerical simulations of an oscillating water column with humidity in the air chamber," Journal of Cleaner Production, vol. 238, Article ID 117898, 2019.

[43] F. Ohle and H. Eckelmann, "Modeling of a von Kármán vortex street at low Reynolds numbers," Physics of Fluids A: Fluid Dynamics, vol. 4, no. 8, pp. 1707-1714, 1992.

[44] A. Roshko, "On the Development of Turbulent Wakes from Vortex Streets," National Advisory Committee for Aeronautics, Washington, DC, USA, ROSnacarpt1191, 1954.

[45] A. Elhanafi, G. Macfarlane, A. Fleming, and Z. Leong, "Experimental and numerical measurements of wave forces on a 3D offshore stationary OWC wave energy converter," Ocean Engineering, vol. 144, pp. 98-117, 2017.

[46] U. Izquierdo, G. A. Esteban, J. M. Blanco, I. Albaina, and A. Peña, "Experimental validation of a CFD model using a narrow wave flume," Applied Ocean Research, vol. 86, pp. 112, 2019.

[47] E. Medina-López, A. Moñino, R. J. Bergillos, M. Clavero, and M. Ortega-Sánchez, "Oscillating water column performance under the influence of storm development," Energy, vol. 166, pp. 765-774, 2019.

[48] X. An, B. Song, W. Tian, and C. Ma, "Numerical simulation of vortex induced piezoelectric energy converter (vipec) based on coupled fluid, structure and piezoelectric interaction," in Proceedings of the OCEANS 2017-Aberdeen, IEEE, Aberdeen, UK, June 2017. 